\title{
PLANIFICACIÓN CONCURRENTE: UNA ALTERNATIVA PARA MEJORAR EL SISTEMA DE PROTECCIÓN DE MENORES ESPAÑOL
}

\section{THE CONCURRENT PLANNING: ALTERNATIVE TO IMPROVE THE SPANISH CHILD PROTECTION SYSTEM}

\author{
BLANCA GÓMEZ BENGOECHEA ${ }^{1}$
}

Recibido: 10/04/2019

Aceptado: 01/07/2019

SUMARIO: I. Introducción. II. Características principales. 1. Concepto. 2. Objetivo. 3. Necesidad de estabilidad: las consecuencias para los niños de la falta de permanencia. 4. Punto de partida. 5. Origen. III. Principios fundamentales. IV. Participantes. 1. Los niños necesitados de protección. 2. Las familias acogedoras/adoptivas. 3. La familia biológica. V. Ventajas e inconvenientes. VI. Razones para introducir la Planificación Concurrente en España.

SUMMARY: I. Introduction. II. Main characteristics. 1. Definition. 2. Objective. 3. Need for stability: the consequences for children of the lack of permanence. 4. Starting point. 5. Origin. III. Fundamental principles. IV. Participants. 1. Children in need of protection. 2. Foster/adopt families. 3. Biological family. V. Advantages and disadvantages. VI. Reasons to introduce the Concurrent Planning in Spain.

Resumen: El sistema de protección de menores español se encuentra, desde la aprobación de las nuevas leyes sobre esta materia en 2015, inmerso en una etapa de reflexión y cambio. La nueva legislación aporta importantes novedades en el campo que regula, pero, a pesar de llevar más de tres años vigente, está todavía pendiente de concretarse en muchas cuestiones.

En este contexto, es necesario pararse a reflexionar sobre la posibilidad de incorporar a la práctica de la protección de menores en España, buenas prácticas y nuevos modelos que ayuden a dar el cambio necesario que pretende propiciar la nueva legislación: lograr para los niños soluciones familiares, estables y consensuadas.

Una de estas prácticas con cierta trayectoria en su aplicación en otros países, es el "Concurrent Planning” o Planificación Concurrente. A lo largo de estas páginas reflexionaremos sobre la naturaleza de este tipo de intervención, sus características, requerimientos y resultados, y su posible aplicabilidad al sistema de protección español.

Palabras clave: protección de menores, adopción, acogimiento, planificación para la permanencia

\footnotetext{
1 Dra. en Derecho. Investigadora Propia Adjunta. Instituto Universitario de la Familia. Universidad Pontificia Comillas. E-mail: bgomez@comillas.edu. ORCID: 0000-0003-2574-7947.
} 
Abstract: Spanish child protection system is, since the adoption of a new regulation on this matter in 2015, immersed in a phase of reflection and change. The new legislation brings important novelties in this field, but, despite having more than three years in force, it is still pending to materialize on many issues.

In this context, it is necessary to think on the possibility of incorporating good practices and new models that could help to achieve family, permanent and consensual solutions for children, as it is demanded by the law.

One of these practices, frequently used in other countries, is the Concurrent Planning. Throughout these article, we will reflect on the nature of this type of intervention, its characteristics, requirements and results, and its possible applicability to the Spanish child protection system.

Keywords: children welfare, adoption, fostering, concurrent planning

\section{INTRODUCCIÓN}

El sistema de protección de menores español se encuentra, desde la aprobación de las nuevas leyes sobre esta materia en el año 2015, inmerso en una etapa de reflexión y cambio. La nueva legislación aporta importantes novedades en el campo que regula, pero, a pesar de llevar más de tres años vigente, está todavía pendiente de concretarse en muchas cuestiones.

Aunque la revisión acerca de los cambios necesarios se ha iniciado en algunas Comunidades Autónomas, varios años después, la normativa de casi todas ellas sigue sin estar adaptada a lo que dispone la nueva legislación, y la transformación que buscaba traer consigo la ley está siendo lenta y difícil.

En este contexto, es necesario pararse a reflexionar sobre la posibilidad de incorporar a la práctica de la protección de menores en España, buenas prácticas y nuevos modelos que ayuden a dar el cambio necesario que pretende propiciar la nueva legislación: lograr para los niños soluciones familiares, estables y consensuadas.

Una de estas prácticas, novedosa para nuestro sistema de protección, pero introducida con éxito en otros países, es el Concurrent Planning. Esta forma de intervención ha sido utilizada en estados en los que la situación respecto a los menores desprotegidos era parecida a la que existe en España (muchos niños en protección, con medidas supuestamente temporales que acaban alargándose durante muchos años, con pocos niños adoptables y pocas familias dispuestas a acoger, pero muchas dispuestas a adoptar...), y puede servir de inspiración en el trabajo por la mejora de la situación de los menores en protección en nuestro país.

A lo largo de estas páginas reflexionaremos sobre la naturaleza de este tipo de intervención, sus características, requerimientos y resultados, y su posible aplicabilidad al sistema de protección español. 


\section{CARACTERÍSTICAS PRINCIPALES}

\section{Concepto}

La Planificación Concurrente es un modo de intervenir con niños en situación de desprotección caracterizado, fundamentalmente, por que, en el momento en que el menor es separado de su familia, se inician a la vez, como procesos simultáneos:

- El trabajo con la familia de origen para lograr el retorno

- La búsqueda y planificación de un recurso alternativo de cuidado familiar permanente, por si el retorno no fuera posible (adopción normalmente, pero también tutela por un familiar o vida independiente supervisada), que se hace cargo del cuidado del niño desde que éste sale de casa.

De esta manera, lo que generalmente se hace de forma secuencial (primero se intenta el retorno, y comprobado que éste no es posible, se busca una alternativa), se inicia de forma simultánea, con la intención de lograr acortar los plazos de incertidumbre y dar estabilidad a los niños.

Este proceso implica, por tanto, la existencia de una sola familia y dos posibles resultados para el menor (la reunificación o la adopción), y se caracteriza por:

- El desarrollo de dos planes concurrentes, de forma que, si la reunificación no es posible, la forma de cuidado ya iniciada está disponible para convertirse en la definitiva.

- El compromiso con la familia, y la revelación desde el inicio, del procedimiento y la metodología de intervención a la familia de origen.

- La comunicación abierta y el contacto frecuente con los padres biológicos.

- La evaluación temprana del pronóstico respecto a una posible reunificación familiar.

- La planificación específica del caso, con la intención de lograr la permanencia en el cuidado en un plazo razonable ${ }^{2}$.

Este inicio de un doble procedimiento se hace con el conocimiento de todos los implicados y seleccionando, para hacerse cargo de los niños, familias dispuestas a asumir el cuidado temporal de los menores mientras se procura que sus familias de origen se puedan volver a hacer cargo de ellos, pero resueltas también a convertirse en su familia definitiva en caso de que el retorno no fuera posible. De modo que se precisa de familias decididas y preparadas para dos posibles resultados diferentes.

Esto supone crear una situación en la que los niños disfrutan de una acogida familiar estable con visitas regulares con su familia de origen hasta que regresen con ella, o, si finalmente no es posible el retorno y son adoptados, habrán estado con su

\footnotetext{
${ }^{2}$ ADOPTUSKIDS, National Resource Center for Diligent Recruitment, "Concurrent Planning. Partnering for Permanency", recuperado el 7 de marzo de 2019, en http://www.nrcdr.org/_assets/files/DRGrantees/year-two-2/CP-PartneringPermanency-NM.pdf; TREGEAGLE, S., MOGGACH, L., COX, E., VOIGT, KL., "A pathway from long foster-care to adoption: findings from an Australian permanency programme", Adoption and Fostering, 2014 38: 115, British Association for Adoption and Fostering (BAAF), p. 118.
} 
familia adoptiva desde el primer momento posible, sin interrupciones ni rupturas de vínculos ya creados ${ }^{3}$.

\section{Objetivo}

El procedimiento parte de la "redefinición" del objetivo principal de la entrada del niño en el sistema de protección: el propósito fundamental es lograr la permanencia en el cuidado lo antes posible. De modo que se "recoloca” también el lugar que ocupa la reunificación con la familia de origen, que es el primer camino en que se debe plantear conseguir la permanencia, pero es sólo una de las varias formas posibles de lograrla de una manera satisfactoria ${ }^{4}$.

En este contexto, la permanencia se define como el marco de condiciones emocionales, físicas y legales que permiten a un niño tener sentido de la seguridad, continuidad, compromiso e identidad ${ }^{5}$ :

- La permanencia física proporciona un entorno estable, en una comunidad conocida y familiar para el niño, en la que sus necesidades son satisfechas.

- La permanencia psicológica tiene que ver con la posibilidad de que el niño se sienta vinculado a un adulto que le proporciona una relación estable, segura y afectuosa.

- La permanencia legal supone que el marco normativo pueda proporcionarle seguridad y estabilidad. Implica el reconocimiento de ciertos derechos, y la exigencia de responsabilidades en la toma de decisiones a quienes están a cargo de la protección del menor.

Significa tener un hogar con la misma familia o el mismo grupo de gente, como parte de una misma comunidad y cultura, y con relaciones con continuidad a largo plazo.

Esta metodología no busca proporcionar un camino rápido o un atajo hacia la adopción, sino hacia el cuidado familiar permanente y hacia la toma de decisiones en un plazo de tiempo adecuado para los niños, ya que el retraso en este ámbito tiene para ellos importantes consecuencias involuntarias ${ }^{6}$. En este sentido, las investigaciones

\footnotetext{
3 "Concurrent Planning creates a situation where either they will enjoy a stable foster placement with regular good quality contact with their parent until returned home, or if they are adopted, they will have been in their adoptive family form the earliest opportunity without disruptions and broken attachment". LAWS, S., WILSON, R., RABINDRAKUMAR, S., "Concurrent Planning Study. Interim Report", Coram, july 2012, recuperado el 7 de marzo de 2019, en https://www.coram.org.uk/sites/default/files/resource_files/Concurrent\%20Planning\%20Study\%202012.p df, p. 31.

4 "Permanency is the goal. Reunification is a primary but not only of several acceptable permanency goals", GERALD P. MALLON, D.S.W., "Understanding and Using Concurrent Planning PPT”, National Resource Center for Family Centered Practice and Permanency Planning at The Hunter College of Social Work, New York, recuperado el 7 de marzo de 2019, en https://www.advokids.org/childhood-mentalhealth/concurrent-planning/.

${ }^{5}$ WALTHAM FOREST CHILDREN'S SERVICES, "Permanency Planning Guidance”, en Procedure Manual, recuperado el 7 de marzo de 2019, en http://walthamforestchildcare.proceduresonline.com/chapters/g_perm_plan.html.

6 "It is not a fast track to adoption, but to permanency". GERALD P. MALLON, D.S.W., "Understanding and Using Concurrent Planning PPT”, Op. Cit.

"This is not a fast track to adoption, but to timely permanency", ADOPTUSKIDS, National Resource Center for Diligent Recruitment, "Concurrent Planning...”, op. cit.
} 
muestran que, cuanto mayor es el retraso en la toma de decisiones, y mayor es el número de emplazamientos durante el acogimiento, mayor es el daño posible para la salud mental de los niños y su desarrollo, y menores las posibilidades de encontrar una familia adoptiva para ellos ${ }^{7}$.

Los retrasos en la toma de decisiones tienen como resultado que niños muy pequeños pasen largos periodos de sus vidas sufriendo malos tratos o cuidado negligente hasta que entran en el sistema de protección de menores. Esto, en ocasiones, viene seguido de largos periodos de tiempo bajo medidas de cuidado temporales, en las que los niños establecen vínculos estrechos con cuidadores no definitivos, y experimentan un importante sentimiento de pérdida una vez que se encuentra para ellos una solución permanente ${ }^{8}$.

\section{Necesidad de estabilidad: las consecuencias para los niños de la falta de permanencia}

El objetivo principal es la permanencia y la estabilidad porque ésta es fundamental para que los niños se puedan desarrollar física, emocional e intelectualmente. Necesitan adultos, familias y comunidades capaces de satisfacer sus necesidades en un contexto predecible y a largo plazo, con los que poder establecer vínculos de apego seguros, fundamentales para el desarrollo de su autoestima, sus relaciones con los demás, sus logros escolares, y su éxito en la vida como adultos ${ }^{9}$.

En contraste con estas necesidades, la vida en el sistema de protección muchas veces no es estable, implica cambios de emplazamiento, y lleva consigo una buena dosis de incertidumbre e impredecibilidad, lo que puede jugar en contra de los intereses de los niños y del desarrollo de su potencial para crecer y desarrollarse de una forma sana ${ }^{10}$.

Intervenir de esta forma evita las rupturas en la construcción de un apego seguro, que tantas veces ocurren cuando se confía el cuidado de un bebé con muchas probabilidades de tener que ser adoptado a una familia acogedora para después tener que moverlo a una familia adoptiva.

Se logra, también, mayor seguridad, que se planteen y se examinen los plazos de intervención, y que se revisen los tiempos y los criterios de los procesos de toma de decisiones. Además, un planteamiento de este tipo favorece dar a las familias la oportunidad de solucionar sus problemas y reconstruirse desde sus fortalezas, e implementar los cambios necesarios para cuidar adecuadamente de sus hijos. Y permite

\footnotetext{
"Harm that can occur to children as an unintended consequence of the time taken to arrive at decisions in care proceedings”, LAWS, S., WILSON, R., RABINDRAKUMAR, S., “Concurrent Planning Study.... Op. Cit., p. 24.

${ }^{7}$ LAWS, S., WILSON, R., RABINDRAKUMAR, S., “Concurrent Planning Study..., Op. Cit., p. 7.

${ }^{8}$ LAWS, S., WILSON, R., RABINDRAKUMAR, S., “Concurrent Planning Study..., Op. Cit., pp. $15,7$.

9 WALTHAM FOREST CHILDREN'S SERVICES, "Permanency Planning Guidance”, Op. Cit. MINNESOTA DEPARTMENT OF HUMAN SERVICES, "Practice Guide for Concurrent Permanency Planning”, p. 3, recuperado el 7 de marzo de 2019, en http://www.mncourts.gov/mncourtsgov/media/scao_library/CJI/Benchbook/Ch-_32__Concurrent_Permanency_Planning_-_Appendix_A.pdf

${ }_{10}$ Muchos niños crecidos en acogimiento tienen trayectorias vitales pobres en comparación con la población general, incluyendo bajos logros educativos, sinhogarismo y problemas emocionales. TREGEAGLE, S., MOGGACH, L., COX, E., VOIGT, KL., “A pathway from long foster-care to adoption...”, Op. Cit., p. 117.
} 
honrar el vínculo que une al niño con su familia biológica a través del trabajo intensivo realizado para intentar la reunificación ${ }^{11}$.

\section{Punto de partida}

Un procedimiento de protección de este tipo implica que los adultos acogedores/adoptantes se hacen cargo del cuidado del menor sin saber si va a ser sólo por un tiempo o si va a ser para siempre y el niño va a terminar siendo su hijo.

Precisamente esta incertidumbre, y el lugar en el que se sitúa, es el fundamento de la Planificación Concurrente: está basado en la idea de que los adultos pueden asumir el riesgo de la separación (o posible pérdida) y la ambigüedad de la relación mejor que los niños, y es sobre ellos sobre quien debe colocarse. De esta forma se invierte la carga emocional del proceso de protección, que habitualmente recae sobre los niños, que son quienes soportan las relaciones temporales, las inseguridades, y los cambios en el cuidado.

No se promete a los padres biológicos que los niños retornarán, ni a los acogedores seguridad en su situación, se les hacen promesas a los niños: si no pueden regresar con su familia de origen, se quedarán con la que les ha cuidado desde que fueron separados de ella. Las promesas se hacen a los hijos, no a los padres.

Un planteamiento de este tipo exige, por otra parte, la ruptura de la "tradicional" separación entre adopción y acogimiento, tanto en lo que se refiere a los niños como a las familias que se ofrecen y seleccionan para una u otra medida. Implica la entrada de los "grises" en un sistema de protección que venía admitiendo solo blancos o negros: o quieres convertirte en padre y te ofreces para la adopción, o quieres cuidar temporalmente de un niño sin ninguna expectativa ni deseo de convertirte en su padre/madre, y te ofreces para el acogimiento en alguna de sus modalidades.

\section{Origen}

Esta forma de intervenir fue formulada por primera vez en Chicago, a finales de los años 60 y principios de los 70, por Irmgard Heymann y sus colegas. Aunque no fue etiquetada como "Concurrent Planning” en aquel momento, su trabajo les llevó a concluir que las soluciones permanentes podían lograrse con mayor rapidez si los trabajadores del caso hablaban y discutían abiertamente sobre la permanencia con los padres biológicos desde el momento en que los niños iniciaban el acogimiento, y si se evaluaban los patrones de comportamiento observados en las visitas y contactos paternos $^{12}$.

A lo largo de los años 70, en otras comunidades de Estados Unidos, los trabajadores sociales del ámbito de la protección de menores comenzaron a hacer acogimientos pre-adoptivos o con cierto riesgo legal cuando la reunificación parecía difícil de conseguir. Después de la aprobación de la Adoption Assistance and Child Welfare Act de 1980, un número considerable de estados desarrollaron planes de

\footnotetext{
${ }^{11}$ SOCIAL SERVICES AGENCY, DEPARTMENT OF FAMILY AND CHILDREN'S SERVICES, County of Santa Clara (California), "Handbook 11, Concurrent Planning and Adoption”, recuperado el 7 de marzo de 2019, en https://www.sccgov.org/sites/ssa/dfcs/opp2/11_adoption/11-1.html\#points.

${ }^{12}$ NATIONAL CENTER FOR CHILD WELFARE EXCELLENCE AT THE SILVERMAN SCHOOL OF SOCIAL WORK, “A Web-based Concurrent Planning Toolkit”, recuperado el 7 de marzo de 2019, en http://www.nccwe.org/toolkits/concurrent-planning/overview.htm
} 
permanencia, aunque muchos de ellos contemplaban la intervención secuencial y no simultánea: primero se intentaba la reunificación con la familia biológica y, si los esfuerzos por lograrla no daban fruto, se exploraban otras opciones de cuidado permanente.

Finalmente, a principios de los años 80, el Departamento de Servicios Sociales de Washington, en colaboración con Linda Katz, de los Servicios Sociales Luteranos, enormemente preocupados por el número de niños pequeños que parecían quedar "atrapados" en el acogimiento, moviéndose de un recurso de acogida a otro, acuñaron el término "Concurrent Planning”. Su metodología de trabajo reconocía que, en casos en los que el pronóstico de reunificación fuera negativo, para niños pequeños, era importante encomendar el cuidado de los niños, lo antes posible, a familias que podrían ser sus familias definitivas si la reunificación no resultaba exitosa. Desde entonces, en las décadas posteriores, otras muchas comunidades han implementado el uso de este modo de planificación para la permanencia ${ }^{13}$.

Más adelante, a finales de los 90, la aprobación de la Adoption and Safe Families Act (1997) tuvo un importante impacto en la aplicación de esta metodología en Estados Unidos, y estableció un contexto en el que esta forma de proteger a los niños comenzó a ser vista de otro modo. Empezó a utilizarse para cumplir con las exigencias de plazos para dotar de medidas definitivas y estables que introducía la ley, y fue reconocida como "buena práctica"14.

\section{PRINCIPIOS FUNDAMENTALES}

Esta forma de intervenir tiene como principios fundamentales los siguientes ${ }^{15}$ :

\footnotetext{
${ }^{13}$ NATIONAL CENTER FOR CHILD WELFARE EXCELLENCE AT THE SILVERMAN SCHOOL OF SOCIAL WORK, "A Web-based Concurrent Planning Toolkit", Op. Cit.; SCHENE, P., "Implementing Concurrent Planning. A Handbook for Child Welfare Administrators", National Welfare Resource Center for Organizational Improvement, Institute for Child and Family Policy, University of Southern Maine, p. 2-3, 2001, recuperado el 7 de marzo de 2019, en http://muskie.usm.maine.edu/helpkids/rcpdfs/concurrent.pdf.

${ }^{14}$ CHILD WELFARE INFORMATION GATEWAY, Concurrent Planning for Permanency for Children, November 2012, p. 2, recuperado el 7 de marzo de 2019, en https://www.childwelfare.gov/pubPDFs/concurrent.pdf\#page=1\&view=Introduction.

Esta ley recoge algunas circunstancias en las que se deben iniciar, inmediatamente, los procedimientos de privación de derechos parentales, y define plazos claros para perseguir la terminación de estos derechos en general, salvo excepciones o casos en los que existan razones de peso para no hacerlo. Además, la ley contempla el Concurrent Planning como medio para lograr el cuidado permanente con mayor rapidez. Para poder cumplir con los plazos previstos en la ley, los estados comenzaron a aplicar esta metodología, que es obligatoria en algunos territorios. NATIONAL CENTER FOR CHILD WELFARE EXCELLENCE AT THE SILVERMAN SCHOOL OF SOCIAL WORK, "A Web-based Concurrent Planning Toolkit”, Op. Cit.

MINNESOTA DEPARTMENT OF HUMAN SERVICES, "Practice Guide for Concurrent Permanency Planning", Op. Cit., p.1.

BOHNSACK, M., CONNELLY, M., "Concurrent Planning: Literature Review and Practice Recommendations,” 2009, California Social Work Education Center, School of Social Welfare, University of California, Berkeley, p. 1.

${ }^{14}$ CHILD WELFARE INFORMATION GATEWAY, Concurrent Planning for Permanency for Children, Op. Cit., p. 2.

${ }^{15}$ SCHENE, P., "Implementing Concurrent Planning..., Op. Cit. p. 1-2, 2001.

BOHNSACK, M., CONNELLY, M., “Concurrent Planning: Literature Review..., Op. Cit., pp. 11-12.
} 


\section{a) Diagnóstico y evaluación tempranos, y revisión del pronóstico del caso}

Es importante tener desde el inicio una evaluación de las posibilidades de la reunificación familiar. Para plantear la intervención y la inclusión en una metodología de este tipo es necesario tener claras las fortalezas de la familia biológica y sus necesidades, y establecer desde el principio las condiciones que se deben dar para que el retorno del menor puede producirse.

En el caso de detectarse dependencias, toxicomanías o problemas de salud mental, es preciso consultar a especialistas en estos ámbitos, con el fin de poder contar con un diagnóstico adecuado y planificar las medidas oportunas para ayudar a los padres a hacer los cambios necesarios. En estos casos es importante, también, iniciar la búsqueda de parientes de forma inmediata.

\section{b) Redefinición de lo que se considera exitoso}

En este contexto, el objetivo o el éxito de la intervención es lograr la permanencia: encontrar un hogar seguro y permanente para el niño lo antes posible.

De manera que, la reunificación con la familia biológica no es lo que se persigue, aunque sea el plan "primario". Es una forma más de lograr la permanencia, pero no la única. En este sentido resulta importante tener presente que el derecho del niño a disfrutar de un cuidado y un contexto familiar permanente no es en absoluto inferior, sino, al menos, equivalente al derecho de los padres biológicos a que se produzca el retorno ${ }^{16}$.

\section{c) Existencia de un Plan A y un Plan B}

Como ya se ha explicado, existe un solo objetivo (el cuidado familiar permanente) y dos formas de lograrlo (el retorno con la familia de origen o la permanencia con la familia de cuidado alternativo), que se ponen en marcha a la vez desde el momento en que el niño tiene que ser separado de sus padres biológicos.

El Plan B puede materializarse a través de una adopción, la constitución de una tutela o el cuidado permanente por parte de familiares u otras personas cercanas con las que existan vínculos previos, pero desde la premisa de ser colocado, desde el inicio, con una familia que, si es necesario, puede terminar siendo su familia permanente.

\section{d) Apertura informativa total}

Todos los participantes en el proceso (familia biológica, familia de acogida/adopción, sistema legal) conocen desde el inicio el plan que existe para proteger al menor. Todos ellos son informados de la existencia de un plan de

MINNESOTA DEPARTMENT OF HUMAN SERVICES, "Practice Guide for Concurrent Permanency Planning”, Op. Cit., pp. 3-5.

SOCIAL SERVICES AGENCY, DEPARTMENT OF FAMILY AND CHILDREN'S SERVICES, County of Santa Clara (California), "Handbook 11, Concurrent Planning and Adoption”, Op. Cit.

THE NEW MEXICO COURT IMPROVEMENT PROJECT TASK FORCE, "Concurrent Planning. A Guide for Judges, Attorneys, and Others Working with Children and Families", June 2005, pp. 4-5, recuperado el 7 de marzo de 2019, en http://www.hunter.cuny.edu/socwork/nrcfcpp/downloads/NMConcurrent_Planning.pdf.

NATIONAL CENTER FOR CHILD WELFARE EXCELLENCE AT THE SILVERMAN SCHOOL OF SOCIAL WORK, “A Web-based Concurrent Planning Toolkit”, Op. Cit.

${ }^{16}$ BOHNSACK, M., CONNELLY, M., “Concurrent Planning: Literature Review..., Op. Cit., p. 11. 
permanencia alternativo a la reunificación, que se convertirá en el plan principal si el niño no puede retornar con su familia de origen. Se informa, además, de los plazos existentes para la toma de decisiones sobre la permanencia; plazos que, en algunos casos, están previstos legalmente ${ }^{17}$. La Adoptions and Safe Families Act establece que los Estados deben iniciar trámites para la supresión de los derechos parentales de los niños que hayan estado en acogimiento durante 15 de los últimos 22 meses, y recoge que la revisión de la permanencia debe realizarse cada 12 meses.

En particular los padres biológicos deben ser informados desde el principio, de forma verbal y por escrito, de las consecuencias que puede tener para su hijo la falta de un entorno familiar de cuidado permanente, las consecuencias que puede tener para su relación con él el incumplimiento de los objetivos marcados en el plan de caso, los servicios de apoyo disponibles para ayudarles a hacer los cambios necesarios de cara al posible retorno, y de sus derechos y responsabilidades (entre ellos colaborar en la búsqueda de parientes que podrían ser valorados para hacerse cargo del cuidado del niño, compartir la información relevante sobre las necesidades del menor, participar en la elaboración del plan de caso, o intervenir en los procedimientos judiciales que se puedan plantear).

La familia acogedora/adoptiva debe recibir información por escrito sobre el niño: nombre, sexo y fecha de nacimiento, datos de su familia biológica, raza, religión, antecedentes en el sistema de protección, posibles problemas de salud o de comportamiento, datos de contacto del último lugar en el que el niño estuvo escolarizado, descripción de sus principales fortalezas, medicación, necesidades alimenticias, datos de contacto del último médico que le atendió...

\section{e) Documentación de los casos}

Es importante que los casos sean adecuadamente documentados por los técnicos que intervengan en los mismos. Debe existir constancia escrita de la situación inicial, los planes de intervención programados, objetivos y plazos, y de los progresos realizados.

Contar con documentación escrita actualizada y suficiente es fundamental para realizar evaluaciones periódicas de la situación del menor y la familia, y puede ser de vital importancia a la hora de iniciar procedimientos de privación de derechos parentales, en los casos en los que esto pueda ser necesario.

\section{f) Hechos, no promesas}

Se exigen (y se valoran) de las familias biológicas comportamientos y no promesas. Es decir, los padres biológicos deben avanzar y progresar en la eliminación de las causas que dieron lugar a la separación de los niños, y su forma de actuar será el indicador principal.

Así, la falta de cooperación en el proceso, la falta de continuidad en tratamientos previamente establecidos, y/o de asistencia a reuniones o requerimientos

\footnotetext{
17 Por ejemplo, en Minnesota la revisión de las decisiones sobre permanencia debe producirse a los 6 meses si el niño tiene menos de 8 años, y deben existir decisiones sobre permanencia para todos los niños pasados 12 meses desde su colocación. Habitualmente se examina el progreso realizado a los 6 meses, si la evolución es positiva se continúa manteniendo la reunificación como plan primario y se vuelve a revisar a los 12 meses; si no se aprecia progreso, a los 6 meses se plantea la permanencia alternativa como plan primario.
} 
judiciales, pueden suponer que el plan de permanencia alternativo se convierta en el plan primario de intervención.

\section{g) Acuerdos por escrito}

Todos los acuerdos que se adopten deben ser recogidos por escrito. Que los acuerdos queden plasmados por escrito ayuda a su revisión posterior, pero puede ser especialmente beneficioso para los padres biológicos, porque ayuda a aclarar expectativas y centrarse en los objetivos y tareas requeridos.

Los acuerdos escritos relacionados con el procedimiento de Planificación Concurrente deben, además, contener plazos claros para la toma de decisiones sobre la permanencia, a los que nos hemos referido al mencionar la necesidad de apertura informativa.

\section{h) Visitas con la familia biológica}

Es fundamental que, mientras se desarrollan en paralelo los dos planes posibles para conseguir el cuidado familiar permanente, el niño mantenga visitas frecuentes con sus padres biológicos.

Mantener el contacto es importante para conservar el vínculo de cara a una posible reunificación familiar; les da a los padres la oportunidad de compartir información y poner en práctica nuevas habilidades y comportamientos en relación con sus hijos, adquiridos durante el procedimiento; y permite a los técnicos que trabajan en el caso evaluar el progreso conseguido.

\section{i) Búsqueda, formación y fidelización de familias acogedoras/adoptantes}

Uno de los pilares fundamentales de una intervención de este tipo es la existencia de familias dispuestas, preparadas y evaluadas positivamente tanto para hacerse cargo del cuidado temporal de un menor, como para convertirse en su familia adoptiva, si finalmente el retorno con la familia biológica no es posible en los plazos establecidos.

La búsqueda de estas familias, su formación y acompañamiento son claves a la hora de mantener un programa de Planificación Concurrente.

\section{PARTICIPANTES}

\section{Los niños necesitados de protección}

Para poner en marcha un procedimiento o itinerario de protección de menores en el que se vaya a aplicar el Concurrent Planning, es necesario definir criterios que permitan determinar a qué casos se va a aplicar este planteamiento y cuál es la población a la que va a dirigirse.

A la hora de realizar esta selección o identificación de casos, se han definido y utilizado algunos indicadores, relacionados principalmente con los pronósticos existentes respecto a la reunificación de la familia (y el éxito o fracaso cosechados en las reunificaciones que se han realizado ${ }^{18}$ ), el perfil de los padres, la existencia de malos

\footnotetext{
${ }^{18}$ En la familia concreta o en otras de características similares.
} 
tratos o conductas/entornos de riesgo para los niños, la implicación de los padres en su cuidado, y los recursos disponibles y la necesidad de priorizar su asignación.

Entre ellos se pueden mencionar los siguientes ${ }^{19}$ :

- Historia previa de renuncia voluntaria a derechos parentales en los padres; o padres que han preguntado sobre la posibilidad de renunciar al niño desde la intervención inicial, o lo han pedido en alguna ocasión.

- Enfermedad mental o consumo de sustancias que hace a los padres o cuidadores incapaces para cuidar y proteger al niño. En este sentido, se mencionan expresamente la existencia previa de un tratamiento sin respuesta, y de un patrón anterior de incumplimiento en relación con la medicación o el tratamiento necesarios.

- Padres menores de 16 años sin un sistema de apoyo apropiado, una vez que la posibilidad de buscar un recurso conjunto para padres (generalmente madres) e hijo ha fracasado, debido al comportamiento o la actitud del progenitor menor de edad.

- Padres no identificados o localizados, o poco implicados en el caso, entre ellos aquellos que no visitan a sus hijos o lo hacen con poca frecuencia.

- Historia previa de malos tratos hacia los hijos, abuso sexual, negligencia severa, o casos en los que los niños han sido víctimas de más de un tipo de maltrato.

- Casos en los que existen importantes riesgos en el entorno en el que viven los niños (o vivirían en caso de retornar): los padres mantienen la convivencia con personas que puedan resultar peligrosas para los menores, existen situaciones de violencia doméstica, o condenas penales previas en alguno de los adultos.

- Supuestos en los que ha existido una reunificación previa que ha fracasado.

Teniendo en cuenta estos indicadores, en los distintos lugares en los que la Planificación Concurrente es utilizada, se define la población con la que aplicarlo y se establecen los cauces para hacerlo teniendo en cuenta, principalmente, el tiempo que los niños llevan fuera de casa, su edad, el resultado de las evaluaciones y el pronóstico realizado, y la historia de la familia, llegándose, incluso, a desarrollar herramientas estandarizadas para decidir quiénes entrarán en este tipo de programas ${ }^{20}$.

En relación con la edad de los niños, es frecuente priorizar para el acceso a este tipo de intervención a los más pequeños, por ser los recursos limitados, exigir la Planificación Concurrente una especial dotación de los mismos, y ser niños con los que este tipo de abordaje logra mejores resultados y evita mayores daños ${ }^{21}$.

\footnotetext{
19 SCHENE, P., "Implementing Concurrent Planning... Op. Cit., p. 11-13; INDIANA DEPARTMENT OF CHILD SERVICES, “Child Welfare Manual”, Chapter 5, Section 15, pp. 1-2, recuperado el 7 de marzo de 2019, en https://www.in.gov/dcs/files/5.15\%20Concurrent\%20Planning\%20\%20An\%20Overview.pdf.

${ }^{20}$ Por ejemplo, en Nuevo México se utiliza una lista de diez ítems relacionados con el niño y la historia de la familia, vinculados a posibles malos tratos o negligencia en el cuidado. Si alguno de estos ítems se marca positivamente, se inicia la valoración del caso para la inclusión en el programa de Concurrent Planning. THE NEW MEXICO COURT IMPROVEMENT PROJECT TASK FORCE, “Concurrent Planning. A Guide for Judges..., Op. Cit., p. 6.

${ }^{21}$ Se suele centrar en estos niños porque la investigación ha documentado que cuanto menor es el niño en el momento en que entra en el sistema de protección, mayor es el tiempo que permanece en él.
} 
Así, por ejemplo, en el Estado de Kentucky, se utiliza sólo para recién nacidos abandonados; en California se dirige a niños menores de diez años cuya evaluación muestra escasas posibilidades de reunificación familiar, basándose en la historia de la familia, las relaciones padres-hijos, y los progresos mostrados por los padres ${ }^{22}$.

En Minnesota se pone en marcha con niños menores de ocho años que, de acuerdo con la evaluación realizada en el momento de su entrada en el sistema de protección, parece que continuarán dentro del mismo más de 90 días, o cuando existe una pobre evaluación respecto al pronóstico de los padres. También se emplea para los menores de ocho años que permanecen en el sistema más allá de 90 días, independientemente de los resultados obtenidos en las evaluaciones realizadas a los padres $^{23}$.

Este plazo también se contempla en Washington, donde se aplica a niños pequeños pertenecientes a familias con problemas cronificados. En concreto menores de tres años que llevan bajo el cuidado del sistema de protección 90 días, o niños que han sufrido abuso o cuidado negligente cuyas familias no han respondido al tratamiento y la intervención con anterioridad ${ }^{24}$.

En Connecticut y Florida se realiza una evaluación de la familia cuando los niños llevan seis meses en el sistema de protección. Si en ese momento el pronóstico no apunta a que sea posible una reunificación, se pone en marcha un plan de permanencia ${ }^{25}$.

En el Reino Unido se emplea con niños pequeños con los que desde el inicio se aprecia una alta probabilidad (80\% o mayor) de que vayan a necesitar ser adoptados. Se utiliza, también, en casos en los que, a pesar de haberse identificado importantes riesgos, los padres muestran voluntad de cuidar a sus hijos y desean demostrar que pueden hacer los cambios necesarios para ello ${ }^{26}$.

En cuanto al tiempo que se espera hasta incluir a los niños en este tipo de intervención, es preciso decidirlo teniendo en cuenta que los tiempos de espera no significan lo mismo para todos los niños, que la edad juega un papel importante en esta cuestión, que es necesaria cierta flexibilidad, y que los medios con los que se cuente también son especialmente relevantes para tomar esta decisión ${ }^{27}$. La evaluación de los servicios de apoyo a la reunificación, su disponibilidad y dotación de recursos, son fundamentales para aplicar la Planificación Concurrente de forma justa y eficaz.

\footnotetext{
${ }^{22}$ CHILD WELFARE INFORMATION GATEWAY, Concurrent Planning for Permanency for Children, Op. Cit., p. 3.

${ }^{23}$ MINNESOTA DEPARTMENT OF HUMAN SERVICES, "Practice Guide for Concurrent Permanency Planning”, Op. CIt., p. 2.

${ }^{24}$ SCHENE, P., "Implementing Concurrent Planning..., Op. Cit., pp. 7-8.

${ }^{25}$ CHILD WELFARE INFORMATION GATEWAY, Concurrent Planning for Permanency for Children, Op. Cit., p. 3.

${ }^{26}$ LAWS, S., WILSON, R., RABINDRAKUMAR, S., “Concurrent Planning Study..., Op. Cit. p. 8.

${ }^{27}$ Una de las desventajas del uso del Concurrent Planning está asociada a la interpretación de los plazos como límites absolutos, independientemente del progreso que estén haciendo los padres: "It can be challenging but it is very important to maintain the balance between the judicious use of time limits to ensure that a child does not remain in Foster care unnecessarily and a rote enforcement of time limits in $a$ way that ignores the full picture of the parents'motivation, efforts, incremental progress and a foreseeable reunification". NATIONAL CENTER FOR CHILD WELFARE EXCELLENCE AT THE SILVERMAN SCHOOL OF SOCIAL WORK, “A Web-based Concurrent Planning Toolkit”, Op. Cit.
} 


\section{Las familias acogedoras/adoptivas}

"I think we both knew that concurrency was the better route. Perhaps not for us, but for the child, yes" 28

Para poder utilizar la Planificación Concurrente como forma de proteger y dar estabilidad a los niños que han tenido que ser separados de su familia de origen, es imprescindible poder contar con otras familias dispuestas a asumir lo que este planteamiento lleva consigo.

Familias conocedoras de este doble objetivo simultáneo (el trabajo para el retorno y la posibilidad de otra protección estable y definitiva), que ejercen inicialmente un rol de acogedoras (mientras se decide si es posible la reintegración familiar), pero al mismo tiempo están decididas y preparadas para hacerse cargo del cuidado del niño definitivamente (generalmente a través de su adopción) si, pasado el tiempo previsto, en función de la edad del menor y las circunstancias del caso, la vuelta con la familia de origen no es posible.

Encontrar a este tipo de familias no es fácil, ya que tienen que admitir la inversión de la carga emocional del proceso de protección, que mencionábamos al tratar sobre la filosofía en la que se basa este procedimiento: son ellos quienes tienen que asumir el riesgo emocional que supone vincularse con un niño que podría regresar con su familia biológica después de un tiempo de trabajo para la reunificación, y lo hacen para que no sea el niño quien tenga que hacerlo, ya que el menor no pierde el vínculo con su familia de origen, y no romperá el que establezca con los acogedores si no es para volver con ella. ${ }^{29}$.

Para contar con familias dispuestas a desempeñar esta tarea es necesario informar de esta posibilidad tanto a los potenciales acogedores como a los potenciales adoptantes, incluirla en las charlas, los materiales y los distintos "circuitos" informativos y de captación, explicando su funcionamiento, riesgos y potencialidades.

Quienes se ofrezcan para ser "familias para la planificación concurrente" deben saber que entre sus funciones está la de sostener dos planes de permanencia distintos, el retorno y la adopción, que es necesario mantener el contacto, frecuente y de calidad, con la familia biológica mientras esté abierta la posibilidad del retorno (y, quizá, después también, aunque de otro modo), y que son ellos quienes deben acompañar al niño en este proceso y apoyar la reunificación mientras ésta se esté planteando como plan primario para el menor.

Por tratarse de un proceso distinto, es preciso que quienes se ofrezcan para ser familias acogedoras/adoptivas sean adecuadamente valoradas y seleccionadas, y cuenten con preparación específica, acompañamiento, y con los apoyos económicos y humanos que precisen en cada una de las etapas por las que pasen.

Es necesario, por tanto, que sean personas capaces de manejar sus emociones, de dar prioridad al interés del niño, de construir una relación positiva con la familia

\footnotetext{
28 “Creo que los dos sabíamos que la planificación concurrente era el mejor camino. Quizá no para nosotros, pero sí para el niño”, ADOPTION AND FOSTERING 22:4. Pp. 30-40, Winter 1998/99, citado por BARNARDOS'S NORTH EAST, “Concurrent Planning Service, A new fostering and adoption service for children under two years old”, recuperado el 7 de marzo de 2019, en http://www.senedd.assembly.wales/documents/s10919/Additional\%20information\%20on\%20the\%20Inq uiry\%20into\%20Adoption\%20-\%20Barnardos\%20Cymru.pdf.

${ }^{29}$ Esta dificultad se ha señalado como uno de los obstáculos a sortear para poder realizar intervenciones protectoras de este tipo.
} 
biológica, y de acompañar al menor en el retorno (si se decide) y en las visitas y contactos (mientras se decide).

Las familias que se han embarcado en este tipo de proyecto manifiestan que es necesario tener presente y valorar el riesgo de establecer vínculos con un niño que es posible que después regrese con su familia. Pero mencionan, también, que cuidar de un niño pequeño, recibirle en su primera, y posiblemente única familia, evitar que tenga que moverse varias veces de lugar y de entorno (con todo lo que eso significa a esa edad), poder beneficiarle de alguna manera, y participar de un proceso positivo para el niño, aun cuando después vuelva con su familia, son cuestiones que les ayudaron a decidirse ${ }^{30}$.

Asumen que si el retorno sucede se sentirán mal, y será difícil, pero entienden que, si el niño finalmente retorna con sus padres, será porque es lo mejor para él, y que este camino puede no ser lo mejor para ellos como adultos, pero sí lo es para el niño y sus necesidades ${ }^{31}$.

Con respecto a los contactos y las visitas, algunas familias expresan que al principio la reacción de la familia biológica hacia ellos no fue buena (cuestión que les resultó fácil de comprender, porque querían recuperar a su hijo), pero que después comenzaron a darse cuenta y a apreciar que los acogedores se preocupaban por su hijo, y que le cuidaban bien, y llegaron a expresar que, si no podía volver con ellos, querían que se quedara con la familia acogedora ${ }^{32}$.

\section{La familia biológica}

La Planificación Concurrente contempla como uno de los procesos posibles, y en principio prioritario, para lograr el cuidado familiar estable para el niño, la recuperación de su familia de origen en sus funciones protectoras.

Para que esta recuperación se produzca y el niño pueda retornar, o para poder decidir acerca del plan de cuidado estable alternativo, y que la transición al mismo se realice de la mejor manera posible, es importante que se den algunas condiciones ${ }^{33}$.

En primer lugar, es necesario hacer partícipes a los padres biológicos del proceso y las decisiones que se tomen a lo largo del mismo. Establecer los objetivos en diálogo y de acuerdo con ellos, mantener la comunicación abierta y atender a sus preocupaciones es importante. Es preciso, también, darles feedback de sus progresos, y mantener presente, y afrontar, la ambivalencia del procedimiento.

\footnotetext{
30 "Concurrent planning. Why it worked for us", recuperado el 7 de marzo de 2019, en http://www.caritascare.org.uk/part-one-concurrent-planning-worked-us/; "We adopted our baby through concurrent planning”, recuperado el 7 de marzo de 2019, en http://www.adoptionmatters.org/blog/adopted-baby-concurrent-planning/

31 ADOPTION AND FOSTERING 22:4. Pp. 30-40, Winter 1998/99, citado por BARNARDOS'S NORTH EAST, “Concurrent Planning Service, A new fostering and adoption service for children under two years old”, recuperado el 7 de marzo de 2019, en http://www.senedd.assembly.wales/documents/s10919/Additional\%20information\%20on\%20the\%20Inq uiry\%20into\%20Adoption\%20-\%20Barnardos\%20Cymru.pdf.

32 http://concurrentplanning.org/concurrent-planning-is-the-perfect-fit-for-our-family/

33 SOCIAL SERVICES AGENCY, DEPARTMENT OF FAMILY AND CHILDREN'S SERVICES, County of Santa Clara (California), "Handbook 11, Concurrent Planning and Adoption", Op. Cit. LAWS, S., WILSON, R., RABINDRAKUMAR, S., “Concurrent Planning Study... Op. Cit., p. 10; SCHENE, P., "Implementing Concurrent Planning..., Op. Cit. pp. 17-18; MINNESOTA DEPARTMENT OF HUMAN SERVICES, “Practice Guide for Concurrent Permanency Planning”, Op. Cit., pp. 9-10.
} 
En la intervención que se realice con ellos, se deben plantear cuáles son sus objetivos en relación con sus hijos, dialogar sobre las posibles soluciones a las dificultades que tienen para hacerse cargo de ellos, las estrategias que les han funcionado otras veces, o la forma en la que pueden ayudar a los niños a lograr los objetivos que son importantes para los propios menores. Hay que procurar que, en este camino, tengan presentes sus fortalezas y las de sus hijos.

No se debe perder de vista que el niño es el centro del proceso, y su bienestar el objetivo principal. En este sentido, se debe preguntar a los padres sobre posibles alternativas de cuidado seguro y permanente que permitan al menor conservar sus vínculos con la familia, los amigos y la comunidad en la que vive, y dialogar con ellos en analogía con la necesidad de todos los padres de pensar sobre quién cuidaría de sus hijos si les pasara algo a ellos.

Para poder mantener abiertas las dos posibilidades de cuidado permanente que supone la Planificación Concurrente, también hay que trabajar con los padres biológicos para que no perciban a la familia acogedora como una competidora, sino como alguien que trabaja con ellos para lograr lo mejor para el niño. Es necesario promover y acompañar esta relación, ya que si piensan que todos los implicados están intentando ayudarles a cumplir con su objetivo (familia acogedora/adoptiva, protección de menores, etc.), y finalmente no es posible el retorno de los niños, será más fácil para ellos participar en la elaboración y puesta en marcha de otros planes de permanencia.

Por último, pero como cuestión especialmente relevante, para aplicar la Planificación Concurrente de forma efectiva y justa, es necesario poner al alcance de las familias los medios necesarios para que puedan intentar rehacerse y recuperar sus funciones con respecto a sus hijos en un tiempo que para ellos, los niños, sea razonable. Esto incrementa la responsabilidad de la administración, que debe prever y facilitar servicios y apoyos eficientes para procurar la reunificación, y debe realizar un diagnóstico realista de los servicios existentes y asegurar su disponibilidad.

\section{VENTAJAS E INCONVENIENTES}

Esta forma de procurar protección estable a los niños en situación de desamparo ha demostrado tener algunas ventajas significativas ${ }^{34}$ :

- Logra para ellos soluciones permanentes de cuidado familiar en plazos más cortos (ya sea con la familia biológica o con familia ajena), reduciendo el tiempo que pasan en acogimiento temporal y dentro del sistema de protección.

- Permite el mantenimiento de las relaciones familiares, el aumento en la continuidad de la relación con padres y hermanos (en los casos en los que no se produce el retorno, se suelen constituir adopciones abiertas), y favorece la comunicación entre las dos familias, que trabajan juntas para satisfacer las necesidades del niño.

\footnotetext{
${ }^{34}$ THE NEW MEXICO COURT IMPROVEMENT PROJECT TASK FORCE, “Concurrent Planning. A Guide for Judges..., Op. Cit., p. 7; NATIONAL CENTER FOR CHILD WELFARE EXCELLENCE AT THE SILVERMAN SCHOOL OF SOCIAL WORK, “A Web-based Concurrent Planning Toolkit”, Op. Cit.; SCHENE, P., “Implementing Concurrent Planning..., Op. Cit., pp. 2,3,8.
} 
- Reduce el número de desplazamientos y entornos de acogida, porque la familia que acoge en primer lugar está preparada y dispuesta para ser la definitiva.

- Ayuda a que las familias sean conscientes de la urgencia de lograr la permanencia en el cuidado para el niño. Esto favorece la renuncia voluntaria a los derechos parentales y la constitución de adopciones abiertas en casos en los que la familia biológica tiene serias dificultades para hacerse cargo adecuadamente del niño, lo que hace disminuir los efectos negativos que estas situaciones tienen para los menores.

- Adecuadamente implantada, la Planificación Concurrente permite el acceso temprano a los servicios de apoyo familiar, ya que el plan de trabajo e intervención comienza en cuanto el niño entra en el sistema de protección.

A pesar de que sus ventajas son importantes, $y$ han sido experimentadas en los ordenamientos jurídicos en los que se utiliza este tipo de intervención, ponerla en marcha no es fácil, y su aplicación presenta también algunos inconvenientes.

Entre los riesgos que presenta se encuentran los siguientes ${ }^{35}$ :

- Identificar la Planificación Concurrente con la adopción, y que por este motivo se minimicen los esfuerzos por la reunificación, se preste menos atención a los servicios que necesitan los padres y no se priorice el mantenimiento de las visitas.

- Asumir que las herramientas que se utilicen para la evaluación de los casos puedan predecir de forma infalible el resultado. Esto también puede llevar a minimizar los esfuerzos por la reunificación y a promover menos visitas.

- Invertir en un resultado concreto de los dos posibles que se plantean desde el inicio, en lugar de dejar evolucionar el caso en función de las actuaciones y decisiones de la familia biológica.

- Diseñar planes de intervención no centrados en la familia. Las intervenciones programadas deben comprometer a los padres, y ayudarles a asumir los roles y responsabilidades importantes para mantener el contacto con los niños, o hacer los cambios necesarios para que estos puedan retornar en el plazo previsto.

- Plantear a las familias adoptantes-acogedoras una estimación del "riesgo legal” de retorno. Hay que plantearles tanto la posibilidad del retorno exitoso, como la de convertirse en familia permanente, y transmitirles que su papel es apoyar los esfuerzos para la reunificación mientras continúan preparadas para ser el Plan B para el niño. Esto exige un apoyo especial, ya que estas familias están llamadas a desempeñar un difícil papel: acompañar los esfuerzos por la reunificación mientras cuidan a unos niños a los que estarían dispuestos a adoptar.

- Pueden producirse importantes resistencias cuando, tras un acogimiento largo en una Planificación Concurrente, se plantea su terminación y la reincorporación, contra todo pronóstico, con la familia biológica.

- Interpretar los plazos como límites absolutos, independientemente de los progresos hechos por los padres. Es necesario mantener el equilibrio entre que el niño permanezca en acogimiento innecesariamente y la aplicación estricta

35 NATIONAL CENTER FOR CHILD WELFARE EXCELLENCE AT THE SILVERMAN SCHOOL OF SOCIAL WORK, “A Web-based Concurrent Planning Toolkit”, Op. Cit. 
de los límites temporales, ignorando la visión general del esfuerzo de los padres, su motivación, progreso y posible reunificación.

Con respecto a los obstáculos en su aplicación, están relacionados, principalmente, con la dificultad para encontrar familias dispuestas a desempeñar este papel, la necesidad de dotar al programa de recursos suficientes, y con la resistencia encontrada, en muchos casos, en los técnicos que tenían que ponerlo en marcha y acompañarlo.

Los esfuerzos por la reunificación requieren de un uso intensivo de recursos y un apoyo especializado: se debería poder contar con dos trabajadores por caso o uno con un reducido número de casos a su cargo, y es preciso buscar familias dispuestas, acompañar a padres y cuidadores, y a los propios niños. Para que este sistema funcione es necesario adecuar la ratio técnicos/número de familias, disponer para ellos una formación y preparación adecuadas, y prever apoyo y supervisión, durante y después del acogimiento, independientemente de cuál sea el resultado ${ }^{36}$.

En cuanto al papel que juegan los técnicos de protección, en muchos casos se han mostrado reacios a iniciar intervenciones en el marco de la Planificación Concurrente, por las incertidumbres que supone para todos los implicados, y porque existe un fuerte compromiso hacia la reunificación de los niños con sus familias biológicas, que tradicionalmente ha llevado a tratar de evitar las adopciones en los casos en los que no existe consentimiento parental. Aunque este enfoque podría ser apropiado cuando niños muy pequeños entran en el sistema de protección, es necesario establecer plazos para la toma de decisiones basados en la edad de los niños, sus necesidades y momento de desarrollo, porque la permanencia en acogimiento temporal puede suponer para ellos daños importantes ${ }^{37}$.

Para tratar de salvar estos obstáculos es necesario que los servicios de protección cuenten, para poner en marcha este tipo de intervenciones, con recursos suficientes y con profesionales con una fuerte capacidad de liderazgo.

\section{RAZONES PARA INTRODUCIR LA PLANIFICACIÓN CONCURRENTE EN ESPAÑA}

Una vez expuestas sus características fundamentales, el modo de funcionar del Concurrent Planning, y la forma en la que se aplica en otros países, cabe preguntarse si una intervención de este tipo podría ayudar a mejorar la vida de los niños necesitados de protección en España.

Como mencionábamos en la introducción de este artículo, la situación del sistema de protección español es parecida a la que tenían en algunos de los lugares en los que la Planificación Concurrente se ha convertido en una práctica frecuente ${ }^{38}$ :

- En nuestro país hay muchos menores con necesidad de protección: más de 40.000 si contamos a los tutelados, los confiados en guarda por sus padres a la administración y los que están en estudio o sujetos a medidas de apoyo previas

\footnotetext{
${ }^{36}$ LAWS, S., WILSON, R., RABINDRAKUMAR, S., “Concurrent Planning Study..., Op. Cit, p. 7; BOHNSACK, M., CONNELLY, M., “Concurrent Planning: Literature Review..., Op. Cit., p.6.

37 TREGEAGLE, S., MOGGACH, L., COX, E., VOIGT, KL., “A pathway from long foster-care to adoption...”, Op. Cit., p. 117.

38 GÓMEZ BENGOECHEA, B., “Aplicación de la nueva legislación de protección de menores: ¿una oportunidad perdida?”, Actualidad Civil, n. ${ }^{0}$ 12, diciembre 2018, No 12, 1 de dic. de 2018, pp. 2-3.
} 
a la guarda o la tutela. De todos ellos cerca del 40\% (casi 14.000) vive en centros.

- Es frecuente que estos niños desarrollen una larga trayectoria vital dentro del sistema, y el índice de retorno exitoso con sus familias de origen, después de haber sido sometidos a medidas de protección en principio temporales, es muy bajo: retornan antes de los 18 años únicamente el 13\% de los menores que han pasado por un acogimiento familiar, y un 18\% de quienes han estado en acogimiento residencial. Es, además, relativamente frecuente, que haya retornos no exitosos o nuevos fracasos en la protección por parte de la familia, y se produzcan "reingresos" en los recursos de protección.

- En la mayor parte de los casos los acogimientos adoptan la modalidad de permanentes $\mathrm{y}$, aunque la mayoría de los acogimientos familiares lo son en familia extensa, en los últimos años se ha producido un ligero aumento en los acogimientos en familia ajena.

Con esta situación, y ante la obligación planteada por la legislación aprobada en 2015 de dar a los niños soluciones familiares, estables y consensuadas, nos enfrentamos a la necesidad de reducir las cifras de niños en acogimiento residencial, reducir los tiempos que los menores pasan sujetos a medidas de protección inicialmente temporales (con la incertidumbre que esto genera respecto a su cuidado), y agilizar los procesos de toma de decisiones.

Quizá, ante esta situación y con estos objetivos, sea conveniente aprovechar lo que se ha hecho o se está haciendo con éxito en otros lugares poniendo en marcha modelos de intervención como el que hemos descrito a lo largo de este artículo.

Para ello habría que comenzar por romper las separaciones absolutas entre adopción y acogimiento con las que se ha funcionado desde hace décadas, abriendo la puerta a ofrecimientos y soluciones en las que quepan planteamientos más flexibles (como ya ocurre con la adopción abierta, regulada por la nueva legislación).

Habría, también, que definir con qué tipo de población querría aplicarse este modelo, y con qué plazos se puede convivir en las esperas de los niños y las oportunidades de recuperación de las familias biológicas.

Sería necesario lanzarse a la búsqueda activa (y no sólo a la espera más o menos pasiva de ofrecimientos) y formación de familias dispuestas a formar parte de procesos de este tipo, y acompañarlas en su tarea.

Y, como pieza fundamental, habría que dar a las familias biológicas verdaderas oportunidades, dotadas de recursos suficientes, para la recuperación de las funciones protectoras de sus hijos.

Es un desafío importante, que supone cambiar planteamientos de intervención muy arraigados, pero entendemos que es necesario y que sería un complemento (que no un sustitutivo) importante de las medidas y formas de proteger ya existentes, que parece que no llegan a ser eficaces en muchos de los casos a los que se enfrenta el sistema.

\section{BIBLIOGRAFÍA}

ADOPTION AND FOSTERING 22:4. Pp. 30-40, Winter 1998/99, citado por BARNARDOS'S NORTH EAST, "Concurrent Planning Service, A new fostering and adoption service for children under two uears old”, 
http://www.senedd.assembly.wales/documents/s10919/Additional\%20information\%20o n\%20the\%20Inquiry\%20into\%20Adoption\%20-\%20Barnardos\%20Cymru.pdf.

ADOPTUSKIDS, National Resource Center for Diligent Recruitment, "Concurrent Planning. Partnering for Permanency", http://www.nrcdr.org/_assets/files/DR-Grantees/year-two-2/CP-PartneringPermanencyNM.pdf.

BOHNSACK, M., CONNELLY, M., "Concurrent Planning: Literature Review and Practice Recommendations,” 2009, California Social Work Education Center, School of Social Welfare, University of California, Berkeley.

CHILD WELFARE INFORMATION GATEWAY, Concurrent Planning for $\begin{array}{llll}\text { Permanency for Children, 2012, } & \text { November }\end{array}$ https://www.childwelfare.gov/pubPDFs/concurrent.pdf\#page=1\&view=Introduction.

GERALD P. MALLON, D.S.W., "Understanding and Using Concurrent Planning PPT", National Resource Center for Family Centered Practice and Permanency Planning at The Hunter College of Social Work, New York, https://www.advokids.org/childhood-mental-health/concurrent-planning/.

GÓMEZ BENGOECHEA, B., “Aplicación de la nueva legislación de protección de menores: ¿una oportunidad perdida?”, Actualidad Civil, n. ${ }^{\circ} 12$, diciembre 2018, $\mathrm{N}^{\mathrm{o}}$ 12, 1 de dic. de 2018.

INDIANA DEPARTMENT OF CHILD SERVICES, “Child Welfare Manual”, Chapter 5 , Section

15 , https://www.in.gov/dcs/files/5.15\%20Concurrent\%20Planning\%20\%20An\%20Overview.pdf.

LAWS, S., WILSON, R., RABINDRAKUMAR, S., "Concurrent Planning Study. Interim Report”, Coram, july 2012. https://www.coram.org.uk/sites/default/files/resource_files/Concurrent\%20Planning\%2 0Study\%202012.pdf.

MINNESOTA DEPARTMENT OF HUMAN SERVICES, "Practice Guide for Concurrent Permanency Planning", http://www.mncourts.gov/mncourtsgov/media/scao_library/CJI/Benchbook/Ch-_32__Concurrent_Permanency_Planning_-_Appendix_A.pdf.

NATIONAL CENTER FOR CHILD WELFARE EXCELLENCE AT THE SILVERMAN SCHOOL OF SOCIAL WORK, "A Web-based Concurrent Planning Toolkit”, http://www.nccwe.org/toolkits/concurrent-planning/overview.htm

SCHENE, P., "Implementing Concurrent Planning. A Handbook for Child Welfare Administrators”, National Welfare Resource Center for Organizational Improvement, Institute for Child and Family Policy, University of Southern Maine, 2001, http://muskie.usm.maine.edu/helpkids/rcpdfs/concurrent.pdf.

SOCIAL SERVICES AGENCY, DEPARTMENT OF FAMILY AND CHILDREN'S SERVICES, County of Santa Clara (California), "Handbook 11, Concurrent Planning and Adoption”, https://www.sccgov.org/sites/ssa/dfcs/opp2/11_adoption/11-1.html\#points.

THE NEW MEXICO COURT IMPROVEMENT PROJECT TASK FORCE, "Concurrent Planning. A Guide for Judges, Attorneys, and Others Working with Children and Families”, June 2005, http://www.hunter.cuny.edu/socwork/nrcfcpp/downloads/NMConcurrent_Planning.pdf. 
TREGEAGLE, S., MOGGACH, L., COX, E., VOIGT, KL., “A pathway from long foster-care to adoption: findings from an Australian permanency programme”, Adoption and Fostering, 2014 38: 115, British Association for Adoption and Fostering (BAAF).

WALTHAM FOREST CHILDREN'S SERVICES, "Permanency Planning Guidance", en Procedure Manual, http://walthamforestchildcare.proceduresonline.com/chapters/g_perm_plan.html. 